\title{
Hypercritical Accretion, Induced Gravitational Collapse, and Binary-Driven Hypernovae
}

\author{
Laura Becerra ${ }^{*} b$, Carlo L. Bianco, ${ }^{a b}$ Maxime Enderli, ${ }^{a c}$ Christopher L. Fryer, ${ }^{f}$ Luca \\ Izzo, ${ }^{a b}$ Milos Kovacevic, ${ }^{a c}$ Marco Muccino, ${ }^{a b}$ Ana V. Penacchioni, ${ }^{d e}$ Giovanni B. \\ Pisani, ${ }^{a b}$ Jorge A. Rueda, ${ }^{a b d}$ Remo Ruffini, ${ }^{a b c d}{ }^{\text {Yu Wang }}{ }^{a b}$ and Elena Zaninoni ${ }^{d}$. \\ ${ }^{a}$ Dip. di Fisica, Sapienza Università di Roma, Piazzale Aldo Moro 5, I-00185 Rome, Italy. \\ ${ }^{b}$ ICRANet, Piazza della Repubblica 10, I-65122 Pescara, Italy. \\ ${ }^{c}$ Université de Nice Sophia Antipolis, CEDEX 2, Grand Château Parc Valrose, Nice, France. \\ ${ }^{d}$ ICRANet-Rio, CBPF, Rua Dr. Xavier Sigaud 150, Rio de Janeiro, RJ, 22290-180, Brazil. \\ eINPE, Av. dos Astronautas, 1758, São José dos Campos, SP, 12227-010, Brazil. \\ f CCS-2, Los Alamos National Laboratory, Los Alamos, NM 87545. \\ E-mail: laura.becerraeicra.it
}

\begin{abstract}
The induced gravitational collapse (IGC) paradigm has been successfully applied to the explanation of GRB-SNe. The progenitor is a tight binary system composed of a CO core and a NS companion. The explosion of the SN leads to hypercritical accretion onto the NS companion, which reaches the critical mass, gravitationally collapsing to a $\mathrm{BH}$ with consequent emission of the GRB. The first estimates of this process were based on a simplified model of the binary parameters and the Bondi-Hoyle-Lyttleton accretion rate. We present the first full numerical simulations of the IGC process. We simulate the core-collapse, the SN explosion, and the hydrodynamic evolution of the accreting material falling into the Bondi-Hoyle surface of the NS. For appropriate binary parameters, the IGC occurs in short timescale $10^{2}-10^{3} \mathrm{~s}$ due to the combined action of photon trapping and neutrino cooling near the NS surface. We address the observational features of this process.
\end{abstract}

Swift: 10 Years of Discovery,

2-5 December 2014

La Sapienza University, Rome, Italy

${ }^{*}$ Speaker.

$\dagger$ ME and MK are supported by the Erasmus Mundus Joint Doctorate Program by grant Nos. 2012-1710 and 2013-1471, respectively, from the EACEA of the European Commission. AVP and EZ acknowledge the support by the International Cooperation Program CAPES-ICRANet financed by CAPES-Brazilian Federal Agency for Support and Evaluation of Graduate Education within the Ministry of Education of Brazil. 


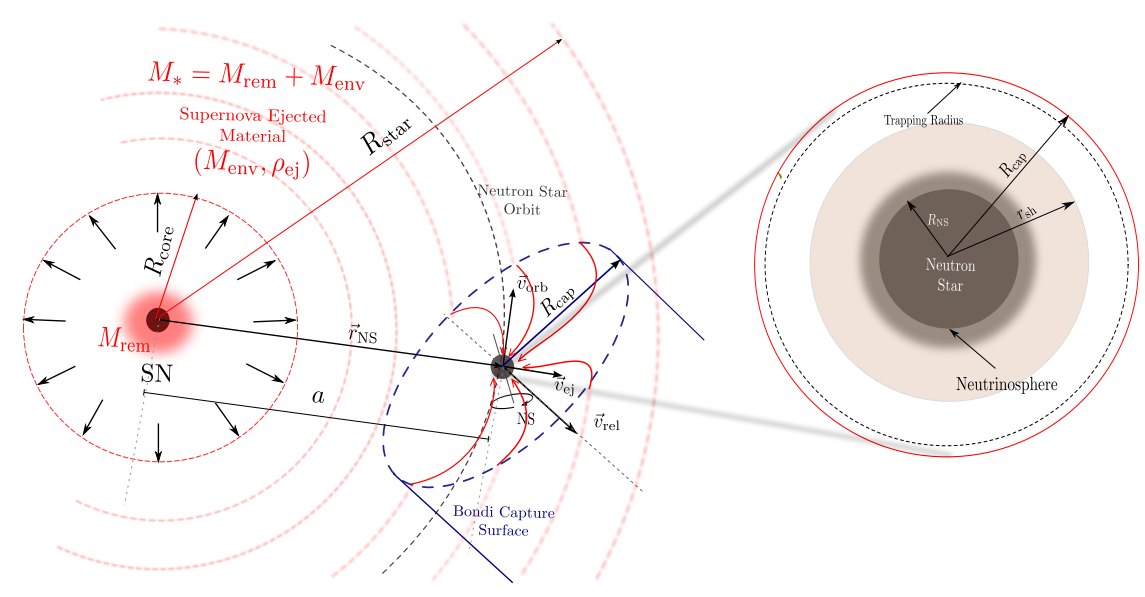

Figure 1: Scheme of the IGC process.

\section{Introduction: Hypercrytical Accretion and Binary-Driven Hypernovae}

The concomitance of a long GRB with a type Ib/c supernova (SN) explosion has been explained through the concept of the induced gravitational collapse (IGC) (Rueda and Ruffini [1], and references therein): the progenitor system is a tight binary system of a carbon-oxygen (CO) star and a neutron star (NS). The core-collapse of the CO core leads to the type $\mathrm{Ib} / \mathrm{c} \mathrm{SN}$ which ejects material that triggers a massive accretion process onto the NS companion. The high accretion rate $\left(\gtrsim 10^{-4} M_{\odot} \mathrm{s}^{-1}\right)$, increases the mass of the NS until it reaches the critical mass value in few seconds. Thus, the NS gravitationally collapse to a black hole $(\mathrm{BH})$ with consequent emission of the GRB (see figure 1).

The theoretical framework of the accretion process in the IGC onto the NS as a function of the binary parameters were first presented in Ref. [1]. The first numerical simulation of the entire process was recently shown in Ref. [2]. We there considered collapsing CO cores leading to $\mathrm{SNe}$ Ic from which we calculate realistic profiles for the density and ejection velocity of the $\mathrm{SN}$ outer layers, essential ingredient for the determination of the accretion rate onto the NS. Once obtained the initial conditions of the material reaching the gravitational capture region of the NS, we followed the hydrodynamic evolution of the accreting material, all the way up to its incorporation onto the NS surface. The trapping of photons inside the Bondi-Hoyle capture region invalids the Eddington accretion limit and thus this process leads to hypercritical accretion rates of up to a few $10^{-2} M_{\odot} \mathrm{s}^{-1}$.

Since its theoretical formulation, the IGC was successfully applied energetic ( $E_{\mathrm{iso}} \gtrsim 10^{52} \mathrm{erg}$ ) GRB-SNe systems, recently called binary-driven hypernovae (BdHNe) [3], which evolve in a rapid sequence lasting a few hundreds of seconds in their rest-frame. These systems are characterized by four distinct episodes, each with specific signatures in its spectrum and luminosity evolution which, up to now, have been verified in several sources with cosmological redshift $z \lesssim 1[4,5,6,7]$, and also in the farthest source, GRB 090423 at $z=8.2$ [8].

In this work we show a simplified model which accounts for all the salient features of BdHNe. This model is in between the initial computation of Ref. [1] and the full numerical simulation of Ref. [2]. We derive the accretion rate onto the NS adopting an improve model of the ejecta density 
with respect to Ref. [1], since we use the envelope density profile of the $\mathrm{CO}$ core prior to explosion, obtained from numerical simulations. However, we do not compute the full dynamics of the $\mathrm{SN}$ explosion as in Ref. [2], but instead we adopt an homologous expansion of the SN ejecta. We simulate the dynamics of the material captured by the NS all the way up $t$ its incorporation into the NS surface by a simplified model of the NS atmosphere and the shock above it, following previous works for the case of fallback onto a NS. Although simplified, we do take account for the dominant cooling mechanisms and the properties of the hot NS atmosphere. We compute the dominant cooling process electron-positron annihilation an use an equation of state for the atmosphere dominated by radiation, electron-positron plasma, and ions. We also explore the general relativistic effects close to the NS surface by calculating the neutrino emission as well as the atmosphere and shock properties in a Schwarzschild background.

\section{Hypercritical Accretion and Infall Region: A Simplified Model}

The hypercritical accretion of the material ejected for the SNe onto the NS can be described through the Bondi-Hoyle-Lyttleton accretion formalism $[9,10,11]$. The rate of accretion, $\dot{M}_{B}(t)$ is given by:

$$
\dot{M}_{B}(t)=\pi \rho_{\mathrm{ej}} R_{\mathrm{cap}}^{2} \sqrt{v_{\mathrm{rel}}^{2}+c_{\mathrm{s}, \mathrm{ej}}^{2}}, \quad \text { with } \quad R_{\mathrm{cap}}(t)=\frac{2 G M_{\mathrm{NS}}(t)}{v_{\mathrm{rel}}^{2}+c_{\mathrm{s}, \mathrm{ej}}^{2}} .
$$

where $G$ is the gravitational constant, $R_{\text {cap }}$ is the capture radius, $M_{\mathrm{NS}}(t)$ the NS mass, $\rho_{\mathrm{ej}}$ and $c_{\mathrm{s}, \mathrm{ej}}$ the density and sound speed of the SN ejecta, $v_{\text {rel }}$ the velocity of the ejecta relative to the NS, $\vec{v}_{\text {rel }}=\vec{v}_{\text {orb }}-\vec{v}_{\text {ej }}$, with $\vec{v}_{\text {ej }}$ the velocity of the SN ejecta and $\left|\vec{v}_{\text {orb }}\right|=\sqrt{\frac{G\left(M_{*}+M_{\mathrm{NS}}\right)}{a}}$ the NS orbital velocity with $a$ the orbital separation, and $M_{*}$ the mass of the pre-SN CO core.

In order to simulate the IGC hypercritical accretion we need to implement a model for the $\mathrm{SN}$-explosion from which we determine the velocity and the density of the SN material near the capture region of the NS. We here adopt a simplified modeling assuming homologous expansion of the SN ejecta, thus the velocity distribution will be of the form $v_{\mathrm{ej}}(r, t)=n r / t$, where $r$ is measured from the SN center, $n$ is called the expansion parameter whose value depends of the hydrodynamical evolution of the ejecta and the circumstellar material $(n=1$ correspond to a free expansion). In the case of homologous expansion, the ejecta density will evolve as $\rho_{\mathrm{ej}}(r, t)=\rho_{\mathrm{ej}}^{0}\left(r / R_{\text {star }}(t), t_{0}\right) \frac{M_{\text {env }}(t)}{M_{\text {env }}(0)}\left(\frac{R_{\text {star }}(0)}{R_{\text {star }}(t)}\right)^{3}$, being $M_{\text {env }}(t)$ the mass ejected in the SN explosion and available to be accreted by the NS, $R_{\text {star }}(t)$ the last layer of the ejected material and $\rho_{\mathrm{ej}}^{0}$ corresponds to the pre-SN density profile.

The evolution of the ejecta density profile is thus related to the structure of the progenitor, prior to the SN explosion. The pre-SN density profile of the progenitor star can be divided in parts: the not ejected material which becomes the central compact remnant, the new NS ( $v$ NS) with mass $M_{\text {rem }} \equiv M_{v \mathrm{NS}}$, approximately the mass of the iron core, and which has almost constant density profile; the material expelled in the SN, namely the CO-envelope, with mass $M_{\text {env }}$. The density profile of the latter can be approximated as a power law: $\rho_{\mathrm{ej}}\left(r, t_{0}\right)=\rho_{\text {core }} r^{-m}$ for $R_{\text {core }}<r \leq R_{0_{\text {star }}}$. The compactness of the $\mathrm{CO}$ core is such that there is no Roche lobe overflow prior to the SN [2]. This condition establish a minimum period for the binary system, $P_{0}$, defined as the one at which the radius of the $\mathrm{CO}$ core equals the Roche lobe size [12]. 
Assuming $c_{\mathrm{s}, \mathrm{ej}} \ll v_{\text {rel }}$ and that the mass of the system remains constant $\left(\dot{M}_{\mathrm{NS}}=-\dot{M}_{\mathrm{env}}=\dot{M}_{B}\right)$, the evolution of the NS accretion rate from (2.1) can be expressed as:

$$
\left(1+\mu_{B}\right)\left(1-\alpha \mu_{B}\right) \dot{\mu}_{B}=\frac{t_{0}}{\tau_{B}} \frac{\tau^{n(m-3)} / \hat{r}^{m}}{\left(1+(\eta \hat{r} / \tau)^{2}\right)^{3 / 2}}, \text { with } \mu_{B} \equiv \frac{M_{B}(\tau)}{M_{0_{\mathrm{NS}}}}, \tau \equiv \frac{t}{t_{0}} \text { and } \hat{r} \equiv 1-\frac{R_{B}}{a}
$$

where $\alpha, \tau_{B}$ and $\eta$ are parameter that depend of the initial conditions of the binary system $(\alpha=$ $\left.M_{0_{\mathrm{NS}}} / M_{0_{\mathrm{env}}}, \eta=n a /\left(t_{0} v_{\mathrm{orb}}\right), \tau_{B}^{-1}=4 \pi G^{2} M_{0_{\mathrm{NS}}} \rho_{\mathrm{ej}}\left(a, t_{0}\right) / v_{\mathrm{orb}}^{3}\right)$. The time $t_{0}$ is defined with the initial velocity of the last layer SNe radius: $v_{\mathrm{Sn}}\left(t_{0}\right)=n R_{0_{\text {star }}} / t_{0}$.

In figure 2 we show the evolution of the Bondi-Hoyle accretion rate onto the NS for different orbital binary periods and an explosion of a $30 M_{\odot}$ zero age main sequence (ZAMS) progenitor star which leads to a $\mathrm{CO}$ core with parameters prior to the explosion (see [2]):

$$
\begin{aligned}
& \rho_{\text {core }}=2.98 \times 10^{8} \mathrm{~g} \mathrm{~cm}^{-3}, \quad m=2.8, \\
& R_{\text {core }}=8.32 \times 10^{7} \mathrm{~cm}, \quad M_{0 \mathrm{env}}=7.94 M_{\odot}, \\
& R_{0_{\text {star }}}=7.65 \times 10^{9} \mathrm{~cm}, \quad M_{v \mathrm{NS}}=1.5 M_{\odot} .
\end{aligned}
$$

During the accretion, the inflowing material shocks as it piles up onto the NS, producing an atmosphere. As the atmosphere compresses, it becomes sufficiently hot to emit neutrinos which cool the infalling material, allowing it to be incorporated into the NS. Thus, the gain of gravitational potential energy due the accretion process is balanced by the emission of neutrinos, produced near the NS surface. In figure 2, for different equations of state for the NS, and in the case of local and global charge neutrality (see [13]), we show the neutrino luminosity considering a spherical steady accretion and general relativistic luminosity $\left(L_{v}=c^{2} \dot{M}\left[\left(1-2 G M_{\mathrm{NS}} /\left(c^{2} R_{\mathrm{NS}}\right)\right)^{-1 / 2}+1\right]\right)$.

In Ref. [14] it was defined the photon trapping radius as the point at which the outward photon diffusion luminosity equates the inward accretion luminosity: $r_{\mathrm{tr}} \approx \kappa \dot{M} /(4 \pi c)$, with $\kappa$ the opacity of the accreted material. For the accretion rates and the opacity of $\mathrm{CO}$ material in the IGC scenario, the trapping radius is larger than the NS radius [2]. Thus, the photons are trapped with the material of the atmosphere above the NS, and the photons emitted inside $r_{\text {tr }}$ are carried inward faster that they diffuse outward. This allows the presence of high accretion rates exceeding the Eddington limit, hence hypercritical.

The accretion produces a shock wave that divides the space into two regions, leading to the SN ejected material make the transition to a subsonic flow $[14,15,16]$. Outside the shock, the infall has negligible pressure and can be described by the free-fall solution. The physics inside the shock front is described by the mass and momentum conservation equations. The spacetime outside the NS is described by the Schwarzchild metric, the gas is assumed polytropic $\left(p \propto \rho^{\gamma}\right)$ and near the NS surface the velocities are subsonic, $(v / c)^{2} \ll 1$.

During the initial phase of the accretion, the shock moves out from the surface of the neutron star towards its steady state position, $r_{\mathrm{sh}}$. With the above assumptions, from the Rankine-Hugoniot jump conditions, through the balance of the neutrino cooling and the accretion energy:

$$
\left(\frac{4 \pi R_{\mathrm{NS}}^{2} \Delta r_{\mathrm{ER}}}{\sqrt{1-\frac{2 G M_{\mathrm{NS}}}{c^{2} R_{\mathrm{NS}}}}}\right) \dot{\varepsilon}_{v}=c^{2} \dot{M}_{B}\left[\left(1-\frac{2 G M_{\mathrm{NS}}}{c^{2} R_{\mathrm{NS}}}\right)^{-1 / 2}-1\right] .
$$



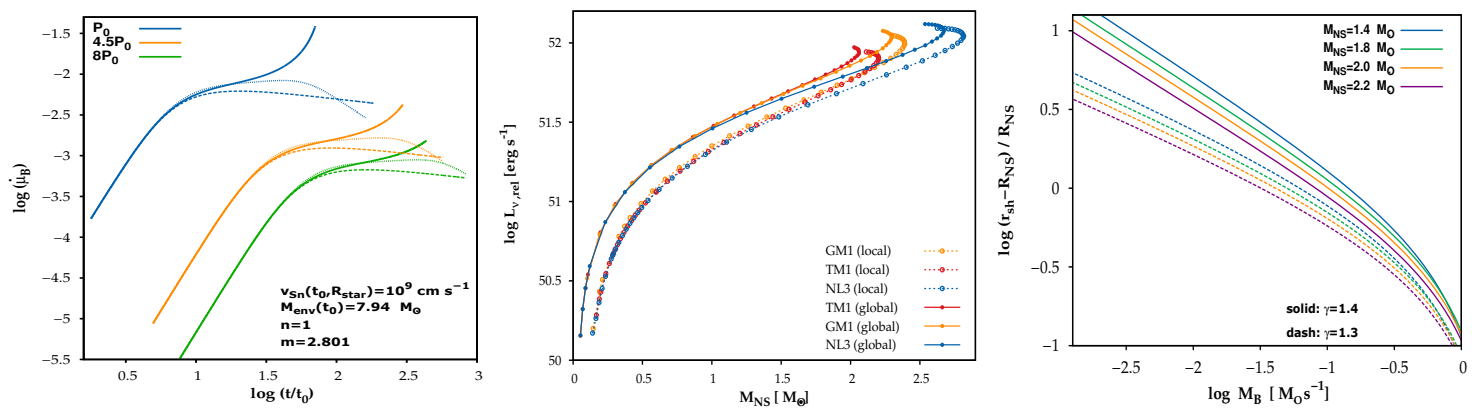

Figure 2: Left panel: hypercritical accretion rate for a $30 M_{\odot}$ supernova progenitor and a neutron star of $1.4 M_{\odot}\left(P_{0}=274.4 \mathrm{~s} \Rightarrow a=1.4 \times 10^{10} \mathrm{~cm}\right)$. Dotted curves: integration at first order of approximation assuming that the NS capture radius does not change with the NS mass; dashed curves: second order approximation assuming that the NS capture radius changes with the NS mass at first order; solid curves: full integration without any approximation. Center panel: relativistic neutrino luminosity ratio. Right panel: thickness of the NS shock envelope.

where have been taken the rate at which gravitational energy is released as the kinetic energy gained in free fall from infinity and consider the proper volume of the cooling region (with $\Delta r_{\mathrm{ER}}$ its thickness) and the proper cooling rate.

The electron-positron annihilation into neutrino pairs $\left(e+e^{-} \rightarrow v+\bar{v}\right)$ dominates the cooling. For a non-degenerate, relativistic and hot plasma, the emission rate of neutrinos by electronpositron annihilation is [17]: $\dot{\varepsilon}_{v}=\dot{\varepsilon}_{\mathrm{e}^{-}} \mathrm{e}^{+}=1.39 \times 10^{25} T_{\mathrm{MeV}}^{9} \mathrm{erg} \mathrm{cm}^{-3} \mathrm{~s}^{-1}$. In figure 2 we show the thickness of the NS shock envelope as a function of the accretion rates and different adiabatic index. The equation of state for the NS atmosphere material correspond to a gas with radiation, non-interacting electrons and positrons and a perfect and completed ionized gas (i.e. the total pressure is $\left.P_{\mathrm{tot}}=P_{\mathrm{rad}}+P_{\mathrm{ion}}+P_{\mathrm{e}^{-}}+P_{\mathrm{e}^{+}}\right)$.

Finally, the post-shock entropy, which can be calculated from the equation of state as $S=$ $\left(E_{\mathrm{sh}}+P_{\mathrm{sh}}-\mu N_{\mathrm{sh}}\right) /\left(\kappa_{B} T\right)$, is a decreasing function of the shock radius position which creates an atmosphere unstable to Rayleigh-Taylor convection during the initial phase of the accretion process. These instabilities can accelerate above the escape velocity driving outflows from the accreting NS with final velocities approaching the speed of light. In the case of fallback material onto the NS in the process of core-collapse $\mathrm{SN}$, it has been shown that these instability might cause the ejection of up to $25 \%$ of the accreting material (see, e.g., Refs. $[18,19]$ ).

The temperature at the base of the atmosphere can be written as $T_{\text {bubble }}=195\left(S R_{\mathrm{NS}_{6}}\right)^{-1}$, with $R_{\mathrm{NS}_{6}}$ the NS radius in units of $10^{6} \mathrm{~cm}$. For the typical hypercritical accretion conditions of BdHNe, the temperature of the bubble when it begins to rise is $T_{\text {bubble }} \sim 5 \mathrm{MeV}$. If it is ejected in a jet, as simulated in [19], it may expand in the lateral direction but not in the radial direction, so $T \propto r^{2 / 3}$. In this scenario, the bubble outflow would have $T_{\text {bubble }} \sim 50 \mathrm{keV}$ at $10^{9} \mathrm{~cm}$ and $T_{\text {bubble }} \sim 15 \mathrm{keV}$ at $6 \times 10^{9} \mathrm{~cm}$. This could explain the temperature and size evolution of the blackbody emitter observed in the early X-ray emission of several BdHNe, see e.g. the case of GRB 090618 [4].

\section{Conclusions}

We have discussed the salient properties of the IGC process leading to BdHNe. We have given 
an estimate of the evolution of the accretion rate onto the NS of the SN ejecta and shown how it might be as large as $10^{-2} M_{\odot} \mathrm{s}^{-1}$ which, in a time of $\sim 10^{2} \mathrm{~s}$, might well bring the NS to its gravitational collapse to a $\mathrm{BH}$ emitting a GRB. We have recalled the time-sequence leading this process and to a high neutrino emission as well as to observable radiation in $\mathrm{X}$ and gamma rays.

\section{References}

[1] J.A. Rueda and R. Ruffini, On the Induced Gravitational Collapse of a Neutron Star to a Black Hole by a Type Ib/c Supernova, ApJL 758 (2012) L7 [arXiv: astro-ph/1206.1684].

[2] C. L. Fryer, J. A. Rueda and R. Ruffini, Hypercritical Accretion, Induced Gravitational Collapse, and Binary-Driven Hypernovae, ApJL 794 (2014) L36 [arXiv : astro-ph/1409.1473].

[3] R. Ruffini, M. Muccini, C.L. Bianco, et al., On binary-driven hypernovae and their nested late X-ray emission, A\&A $\mathbf{5 6 5}$ (2014) L10 [arXiv: astro-ph/1404.3946].

[4] L. Izzo, R. Ruffini, A.V. Penacchioni, et al., A double component in GRB 090618: a proto-black hole and a genuinely long gamma-ray burst, $A \& A \mathbf{5 4 3}$ (2012) A10 [arXiv: 1206.2887 ].

[5] A.V. Penacchioni, R. Ruffini, L. Izzo, et al., Evidence for a proto-black hole and a double astrophysical component in GRB 101023, A\&A 538 (2012) A58 [arXiv: 1112.2970$].$

[6] A.V. Penacchioni, R. Ruffini, C.L. Bianco, et al., GRB 110709B in the induced gravitational collapse paradigm, A\&A 551 (2013) A133 [arXiv:1301.6014].

[7] G.B. Pisani, L. Izzo, R. Ruffini, et al., Novel distance indicator for gamma-ray bursts associated with supernovae, A\&A 552 (2013) L5 [arXiv:astro-ph/1304.1764].

[8] R. Ruffini, L. Izzo, M. Muccini, et al., Induced gravitational collapse at extreme cosmological distances: the case of GRB 090423, A\&A 569 (2014) A39.

[9] F. Hoyle and R.A. Lyttleton, The effect of interstellar matter on climatic variation, in proceedings of the Cambridge Philosophical Society, 1939.

[10] H. Bondi and F. Hoyle, On the mechanism of accretion by stars, MNRAS 104 (1944) 273.

[11] H. Bondi, On spherically symmetrical accretion, MNRAS 112 (1952) 195.

[12] P. P. Eggleton, Approximations to the radii of Roche lobes, ApJ 268 (1983) 368.

[13] R. Belvedere, D. Pugliese, J. A. Rueda, R. Ruffini and S. S. Xue, Neutron star equilibrium configurations within a fully relativistic theory with strong, weak, electromagnetic, and gravitational interactions, Nuclear Physics A $\mathbf{8 8 3}$ (2012) 1.

[14] R. A. Chevalier, Neutron star accretion in a supernova, ApJ 346 (1989) 847.

[15] J. C. Houck and R. A. Chevalier, Steady spherical hypercritical accretion onto neutron stars, ApJ 376 (1991) 234.

[16] C. L. Fryer, W. Benz and M. Herant, The Dynamics and Outcomes of Rapid Infall onto Neutron Stars, ApJ 460 (1996) 801 [arXiv: astro-ph/9509144].

[17] F. G. Yakovlev, A. D. Kaminker,O. Y. Gnedin and P. Haensel, Neutrino emission from neutron stars, ApJ 354 (2001) 1 [arXiv:astro-ph/0012122].

[18] C. L. Fryer, F. Herwig, A. Hungerford and F. X. Timmes, Supernova Fallback: A Possible Site for the r-Process, ApJL 646 (2006) L131 [arXiv:astro-ph/ 0606450 ].

[19] C. L. Fryer, Neutrinos from Fallback onto Newly Formed Neutron Stars, ApJ 699 (2009) 409 [arXiv:astro-ph/0711.0551] 